\title{
Computer-Assisted Sperm Analysis of Freezable and Nonfreezable Mithun (Bos frontalis) Semen
}

\author{
P. Perumal, ${ }^{1}$ S. K. Srivastava, ${ }^{2}$ S. K. Ghosh, ${ }^{2}$ and K. K. Baruah ${ }^{3}$ \\ ${ }^{1}$ Animal Reproduction Laboratory, National Research Centre on Mithun (ICAR), Jharnapani, Nagaland 797 106, India \\ ${ }^{2}$ Animal Reproduction Division, Indian Veterinary Research Institute, Izatnagar, Bareilly, Uttar Uttar Pradesh 243 122, India \\ ${ }^{3}$ Animal Physiology Division, National Research Centre on Mithun (ICAR), Jharnapani, Nagaland 797 106, India
}

Correspondence should be addressed to P. Perumal; perumalponraj@gmail.com

Received 27 June 2014; Revised 31 July 2014; Accepted 4 August 2014; Published 18 August 2014

Academic Editor: Sami Dridi

Copyright (C) 2014 P. Perumal et al. This is an open access article distributed under the Creative Commons Attribution License, which permits unrestricted use, distribution, and reproduction in any medium, provided the original work is properly cited.

\begin{abstract}
The present study was undertaken to assess the motility and velocity parameters of sperm of freezable and nonfreezable ejaculates by computer-assisted sperm analyser (CASA) such as Hamilton-Thorne Semen Analyser IVOS 11 in mithun semen. Fifty ejaculates (twenty-five ejaculates each for freezable and nonfreezable semen ejaculates) were collected from ten matured mithun bulls. CASA parameters, motility parameters such as forward progressive motility (FPM) (\%), nonprogressive motility (NPM) (\%), total motility (TM) (\%), and static sperms (SM) (\%); velocity parameters such as curvilinear velocity (VCL) $(\mu \mathrm{m} / \mathrm{sec})$, straight line velocity (VSL) $(\mu \mathrm{m} / \mathrm{sec})$, average path velocity (VAP) $(\mu \mathrm{m} / \mathrm{sec})$, linearity (LIN) (\%), straightness (STR) (\%), wobble (WOB) (\%), amplitude of lateral head displacement (ALH) $(\mu \mathrm{m})$, and beat/cross-frequency $(\mathrm{BCF})(\mathrm{Hz})$ were measured by CASA analyser. The result revealed that these parameters varied significantly $(P<0.05)$ between the freezable and nonfreezable ejaculates and freezable ejaculates have significantly $(P<0.05)$ higher value than nonfreezable ejaculates. It was concluded that most of the CASA parameters were significantly lower in nonfreezable ejaculates than in freezable ejaculates in mithun and confirmed that the CASA was effective for a quick and objective analysis of motility and velocity parameters in mithun semen.
\end{abstract}

\section{Introduction}

Mithun (Bos frontalis) is a semiwild, free-range, rare bovine species present in the North-Eastern Hill (NEH) region of India and is believed to have originated more than 8000 years ago from wild Indian gaur (Bos gaurus) [1]. The animal has an important place in the social, cultural, religious, and economic life of the tribal population of NEH region. As per the livestock census of India (2007), the mithun population is decreasing gradually due to lack of suitable breeding bulls, increase in intensive inbreeding practices, and lack of suitable breeding management. Greater efforts are required from all quarters to preserve the mithun population to enhance the socioeconomic status of this region. Since mithuns are semiwild animal and not fully domesticated, natural breeding is practiced in this species with accompanied limitations like cost and disease transmission. Thus, use of artificial insemination for improvement of its pedigree is utmost essential.
Etiology for infertility in male animals is varied and complex. Infertility is diagnosed by history, clinical signs, physical examination of external and internal genitalia, assessment of libido and sexual behaviour, semen collection and evaluation, and various hormone analyses related to semen production [2]. Subjective semen analysis is carried out by conventional method such as assessment of sperm concentration, motility, and morphology by phase contrast microscope [3]. However, the assessment of semen quality in terms of motility, velocity, swimming pattern, sperm head behaviour, and so forth, may help in better understanding of the possible sperm function, semen quality, and selection of suitable semen and bulls for cryopreservation in mithun. Various methods are used to estimate sperm motility and velocity parameters, which have ranged from very simple techniques such as timeexposure or multiple exposure photomicrographies to the very sophisticated CASA techniques [4]. CASA system yields accurate, objective assessment, repeatable, and reliable results on different semen parameters such as TM, FPM and different 
velocity parameters [5] based on the measurement of individual sperm cells. Recent reports suggested that CASA does not only measure the proportion of motile spermatozoa but also measures other sperm motion parameters derived from individual sperm cells and it has more predictive power on fertility potential of semen ejaculates [6]. In bovine species, specific motion parameters have been positively correlated with fertility $[7,8]$. In addition to the use of computerized techniques to predict semen fertility, CASA also provides a useful tool to study the effects of various in vitro procedures on sperm motility as well as the means to study the phenomenon of sperm hyperactivation. Spermatozoa FPM along with certain velocity parameters are essential for the spermatozoa to achieve fertilization. Spermatozoa kinematic parameters such as PFM, VSL, VCL, ALH, and LIN were positively correlated with bull fertility $[9,10]$.

A spermatozoon has significantly higher VCL and ALH, indicating that there is major bending of the mid piece and large amplitude of lateral head displacement. This signifies the hyperactivation of the spermatozoa. Hyperactivation in turn implies high energy state of the spermatozoa, which is essential for sperm penetration through cervical mucus, zona pellucida, fuse with the oocytes, and successful fertilization [11]. Spermatozoa motility and velocity parameters reflect their mitochondrial function and energy status indirectly. In bovine, specific motion parameters have been correlated and related to fertility of sperm $[7,9]$.

Study of CASA parameters was reported in domestic animal species such as cattle [10, 12], buffalo [13, 14], sheep [15], goat [16], boar [17], and dog [18]. Further, perusal of literatures revealed that no information on CASA parameters of freezable and nonfreezable ejaculates in mithun species. Hence, the objective of this study was to assess the motility and velocity parameters measured by CASA to pursuit future sperm preservation protocols in mithun.

\section{Material and Methods}

2.1. Experimental Animals. Ten apparently healthy mithun bulls of approximately 3 to 5 yrs of age were selected from the herd in mithun farm, National Research Centre on Mithun (ICAR), Jharnapani, Nagaland, India. The average body weight of these bulls was $501 \mathrm{~kg}$ (493 to $507 \mathrm{~kg}$ ) at 35 yrs of age with good body condition (score 5-6) maintained under uniform feeding, housing, and lighting conditions. The study area lies between $25^{\circ} 54^{\prime} 30^{\prime \prime}$ North latitude and $93^{\circ} 44^{\prime} 15^{\prime \prime}$ East longitude and at an altitude range of 250300 mean sea level. Each experimental animal was fed in this experiment as per the farm schedule. These were offered ad libitum drinking water, $30 \mathrm{~kg}$ mixed jungle forages $(18.4 \%$ dry matter and $10.2 \%$ crude protein), and $4 \mathrm{~kg}$ concentrates (87.1\% dry matter and $14.5 \%$ crude protein) daily fortified with mineral mixture and salt. During the study, all the experimental protocols met the Institutional Animal Care and Use Committee regulations.

2.2. Semen Collection, Incubation, and Evaluation. A total of 50 ejaculates were collected from ten bulls through rectal
TABLE 1: Software settings of HTR IVOS 11 used in the study.

\begin{tabular}{lc}
\hline Parameters & Value \\
\hline Chamber type & Leja 4 \\
Temperature of analysis $\left({ }^{\circ} \mathrm{C}\right)$ & 37.0 \\
Fields acquired & 10 \\
Frame rate $(\mathrm{Hz})$ & 60 \\
Number of frames & 30 \\
Minimum static contrast & 35 \\
Minimum cell size (pixels) & 5 \\
Straightness $(\mathrm{STR})$, thresholds $(\%)$ & 70 \\
VAP cut-off $(\mu \mathrm{m} / \mathrm{s})$ & 30 \\
Progressive minimum VAP $(\mu \mathrm{m} / \mathrm{s})$ & 50 \\
VSL cut-off $(\mu \mathrm{m} / \mathrm{s})$ & 15 \\
Cell intensity & 80 \\
Magnification & 1.89 \\
\hline
\end{tabular}

massage method. Briefly, massage was performed by back and forth hand motion over the ampulla, prostate, and seminal vesicles and then the urethralis muscles were rhythmically stroked [19] followed by the gentle milking of ampullae one by one, which resulted in erection and ejaculation. During collection, the initial transparent secretions were discarded and neat semen drops were collected in a graduated test tube with the help of a funnel. The ejaculates were evaluated and accepted for evaluation if the following criteria were met: concentration: $>500$ million/mL; mass activity: $>3+$, individual motility: $>70 \%$; and total abnormality: $<10 \%$. Immediately after collection, the samples were kept in a water bath at $37^{\circ} \mathrm{C}$ and evaluated for volume, colour, consistency, mass activity, and $\mathrm{pH}$. After the preliminary evaluations, ejaculates were subjected to the initial dilution with prewarmed $\left(37^{\circ} \mathrm{C}\right)$ Tris-citrate extender. The partially diluted samples were then brought to the laboratory in an insulated flask containing warm water $\left(37^{\circ} \mathrm{C}\right)$ for further processing.

2.3. Sample Selection. These ejaculates were split into freezable and nonfreezable ejaculates based on the postthaw motility $[20,21]$. Ejaculates having postthaw motility $40 \%$ and above were considered as freezable ejaculates whereas nonfreezable ejaculates were those having postthaw motility less than $40 \%$.

2.3.1. Computer-Assisted Semen Analysis (CASA). The motility and velocity parameters were evaluated by Hamilton Thorne Sperm Analyser, version IVOS 11 (HTM-IVOS, Version 10.8, Hamilton Thorne Research, Beverly, MA, USA). This CASA system consists of a phase-contrast microscope, camera, minitherm heating stage, image digitizer, and computer for saving and analyzing the data. The software settings are shown in Table 1.

After semen collection, the sperm concentration was first estimated using a phase-contrast microscope (Nikon, Eclipse $80 \mathrm{i}$; $400 \mathrm{x}$ magnification). $25 \mu \mathrm{L}$ of semen was diluted into $50-100 \mu \mathrm{L}$ of Tris (formulated for bull semen) and $5 \mu \mathrm{L}$ of this diluted semen was loaded into a prewarmed dual 
TABLE 2: Mean $( \pm$ SE) motility and velocity parameters of freezable and nonfreezable ejaculates of mithun semen.

\begin{tabular}{lcc}
\hline CASA parameters & Freezable semen $(n=25)$ & Nonfreezable semen $(n=25)$ \\
\hline Forward progressive motility (\%) & $58.95 \pm 3.68^{\mathrm{a}}$ & $38.17 \pm 3.44^{\mathrm{a}}$ \\
Nonprogressive motility (\%) & $17.47 \pm 2.20^{\mathrm{b}}$ & $20.23 \pm 1.83^{\mathrm{a}}$ \\
Total motility (\%) & $76.52 \pm 3.29^{\mathrm{b}}$ & $58.40 \pm 3.45^{\mathrm{a}}$ \\
Static sperms (\%) & $23.58 \pm 3.38^{\mathrm{a}}$ & $41.60 \pm 3.46^{\mathrm{b}}$ \\
Curvilinear velocity (VCL) $(\mu \mathrm{m} / \mathrm{sec})$ & $188.83 \pm 4.65^{\mathrm{b}}$ & $136.67 \pm 4.88^{\mathrm{a}}$ \\
Straight line velocity (VSL) $(\mu \mathrm{m} / \mathrm{sec})$ & $89.77 \pm 3.49^{\mathrm{b}}$ & $60.63 \pm 3.82^{\mathrm{a}}$ \\
Average path velocity (VAP) $(\mu \mathrm{m} / \mathrm{sec})$ & $118.58 \pm 3.72^{\mathrm{b}}$ & $88.45 \pm 3.18^{\mathrm{a}}$ \\
Linearity (LIN) (\%) & $46.94 \pm 1.53^{\mathrm{b}}$ & $43.89 \pm 1.96^{\mathrm{a}}$ \\
Straightness (STR) $\%)$ & $74.81 \pm 2.28^{\mathrm{b}}$ & $67.76 \pm 2.78^{\mathrm{a}}$ \\
Wobble (WOB) $\%)$ & $62.85 \pm 1.45$ & $65.30 \pm 1.98$ \\
Amplitude of lateral head displacement (ALH) $(\mu \mathrm{m})$ & $8.84 \pm 1.18^{\mathrm{b}}$ & $5.18 \pm 1.35^{\mathrm{a}}$ \\
Beat/cross-frequency (BCF) $(\mathrm{Hz})$ & $28.23 \pm 1.70^{\mathrm{b}}$ & $22.54 \pm 1.63^{\mathrm{a}}$ \\
\hline
\end{tabular}

Figures with same superscript $(a, b)$ do not differ significantly in rows.

chamber disposable Leja slide and was allowed to settle on the minitherm heating stage $\left(38^{\circ} \mathrm{C}\right)$ before the analysis.

The following parameters such as percentage of FPM, NPM, TM, SM, VAP, VSL, VCL, ALH, BCF, and LIN were measured. A minimum of 200 spermatozoa from at least two different drops of each sample were analyzed from each specimen. The number of objects incorrectly identified as spermatozoa was manually removed and final analysis was done for each sample.

2.4. Statistical Analysis. The results were analysed statistically and expressed as the mean \pm SEM. Significant difference between the freezable and nonfreezable semen ejaculates were estimated with Student's $t$-test using the SPSS/PC computer program (version 15.0; SPSS, Chicago, IL). Differences with values of $P<0.05$ were considered to be statistically significant after arcsine transformation of percentage data by using SPSS 15. Correlation between the motility and velocity parameters was established with correlation coefficient being done as per Pearson's method. Differences at $P<0.05$ were considered to be statistically significant.

\section{Result and Discussion}

The sperm motility and velocity parameters were evaluated by Hamilton Thorne Sperm Analyser and result was presented in Table 2. The percent of TM and FPM were significantly $(P<0.05)$ higher in freezable ejaculates than in nonfreezable ejaculates and NPM and SM were significantly higher in nonfreezable ejaculates than in freezable ejaculates. Similarly, velocity parameters were significantly $(P<0.05)$ higher in freezable ejaculates than in nonfreezable ejaculates in mithun species. Thus, it may enhance the quality of semen by preserving efficiently during artificial insemination procedure. Similar report was observed in crossbred cattle [10, 22], Bali cattle [23], buffalo [13,14], sheep [15], goat [16], boar [17], and $\operatorname{dog}[18]$.

The assessment of sperm motility using the conventional microscopical methods is difficult and subjective. High variations have been reported for the estimation of motility parameters of the same ejaculates [24]. CASA is an accurate technique used for the assessment of the motility and velocity parameters of mithun semen. High number of spermatozoa can be analysed individually in a short period of time [5].

Compared with the results of report of others, the velocity parameters of mithun bulls were highly varied [9]. Motility and velocity parameters are varied with the factors such as age, time of collection, time between ejaculations, energy stores of sperm, presence of surface acting agents in the cell membrane such as agglutinins and detergents, viscosity, osmolarity, $\mathrm{pH}$, temperature, ionic concentration of seminal plasma, and presence of the mineral elements like $\mathrm{Cu}, \mathrm{Zn}$, $\mathrm{Mn}$, and hormones, prostaglandins, and so forth [25].

The sperm mobility phenotype can be attributed to specific sperm velocity parameters of individual sperm as determined by CASA. The motion parameters such as VSL, LIN, and BCF contribute to the overall sperm motility characters in bulls, as these were all significantly $(P<0.05)$ correlated with sperm mobility. In the present experiment, various types of sperm motility representing VCL, VSL, VAP, LIN, STR, WOB, ALH, and BCF were also significantly higher for the ejaculates classified as freezable compared with the nonfreezable ejaculates. The parameter LIN is a measure of linearity and the BCF motion parameter indicates the number of times the sperm track crosses the smoothed path, both of which indicate linear progression. Thus, spermatozoa in freezable ejaculates swim faster and straighter than did spermatozoa in nonfreezable ejaculates. This may be biologically significant because the sperm mobility phenotype on the basis of research with whole ejaculates is predictive of fertility $[26,27]$.

Semen samples with high FPM and TM had significantly higher positive correlation with velocity parameters in freezable quality semen (Table 3 ). The samples with high PFM had higher VAP, progressive velocity, and track speed. This was similar to the findings of Anil Kumar et al. [13] and Perumal et al. [10] for path velocity. The average path velocity was significantly and positively correlated with progressive velocity, track speed, and ALH. The high positive correlation observed between VAP, VSL, VCL, and ALH, between VSL and VCL, 
TABLE 3: Correlation between the motility and velocity parameters of sperm of freezable semen ejaculates of mithun.

\begin{tabular}{|c|c|c|c|c|c|c|c|c|c|c|c|c|}
\hline & FPM & NPM & TM & SM & VCL & VSL & VAP & LIN & STR & WOB & ALH & $\mathrm{BCF}$ \\
\hline FPM & 1.00 & -0.52 & $0.92^{*}$ & $-0.93^{*}$ & $0.75^{*}$ & $0.85^{*}$ & $0.68^{*}$ & 0.68 & $0.66^{*}$ & -0.40 & $0.67^{*}$ & $0.88^{*}$ \\
\hline NPM & & 1.00 & -0.15 & 0.15 & 0.03 & -0.05 & 0.03 & -0.14 & -0.17 & 0.16 & 0.03 & -0.08 \\
\hline TM & & & 1.00 & $-0.96^{*}$ & $0.86^{*}$ & $0.96^{*}$ & $0.78^{*}$ & $0.72^{*}$ & $0.68^{*}$ & -0.39 & $0.79^{*}$ & $0.97^{*}$ \\
\hline SM & & & & 1.00 & $-0.87^{*}$ & $-0.95^{*}$ & $-0.77^{*}$ & $-0.73^{*}$ & $-0.70^{*}$ & 0.38 & $-0.81^{*}$ & $-0.95^{*}$ \\
\hline VCL & & & & & 1.00 & $0.87^{*}$ & $0.96^{*}$ & 0.34 & 0.33 & -0.23 & $0.96^{*}$ & $0.91^{*}$ \\
\hline VSL & & & & & & 1.00 & $0.78^{*}$ & 0.61 & 0.57 & -0.33 & $0.82^{*}$ & $0.80^{*}$ \\
\hline VAP & & & & & & & 1.00 & 0.24 & 0.14 & 0.02 & $0.98^{*}$ & $0.85^{*}$ \\
\hline LIN & & & & & & & & 1.00 & $0.91^{*}$ & -0.43 & 0.25 & $0.67^{*}$ \\
\hline STR & & & & & & & & & 1.00 & $-0.76^{*}$ & 0.17 & 0.62 \\
\hline WOB & & & & & & & & & & 1.00 & -0.02 & -0.34 \\
\hline ALH & & & & & & & & & & & 1.00 & $0.85^{*}$ \\
\hline BCF & & & & & & & & & & & & 1.00 \\
\hline
\end{tabular}

* Correlation coefficients were significant; $P<0.05$.

TABLE 4: Correlation between the motility and velocity parameters of sperm of nonfreezable semen ejaculates of mithun.

\begin{tabular}{|c|c|c|c|c|c|c|c|c|c|c|c|c|}
\hline & FPM & NPM & TM & SM & VCL & VSL & VAP & LIN & STR & WOB & ALH & $\mathrm{BCF}$ \\
\hline FPM & 1.00 & -0.38 & $0.96^{*}$ & $-0.95^{*}$ & $0.85^{*}$ & $0.90^{*}$ & $0.79^{*}$ & $0.75^{*}$ & $0.90^{*}$ & $-0.82^{*}$ & $0.78^{*}$ & $0.88^{*}$ \\
\hline NPM & & 1.00 & -0.11 & 0.11 & 0.03 & -0.09 & -0.03 & -0.25 & -0.12 & -0.10 & 0.01 & -0.03 \\
\hline $\mathrm{TM}$ & & & 1.00 & $-0.94^{*}$ & $0.92^{*}$ & $0.94^{*}$ & $0.85^{*}$ & $0.73^{*}$ & $0.93^{*}$ & $-0.91^{*}$ & $0.85^{*}$ & $0.94^{*}$ \\
\hline SM & & & & 1.00 & $-0.90^{*}$ & $-0.95^{*}$ & $-0.86^{*}$ & $-0.75^{*}$ & $-0.91^{*}$ & $0.90^{*}$ & $-0.86^{*}$ & $-0.95^{*}$ \\
\hline VCL & & & & & 1.00 & $0.97^{*}$ & $0.98^{*}$ & $0.64^{*}$ & $0.87^{*}$ & $-0.92^{*}$ & $0.97^{*}$ & $0.98^{*}$ \\
\hline VSL & & & & & & 1.00 & $0.94^{*}$ & $0.82^{*}$ & $0.96^{*}$ & $-0.87^{*}$ & $0.93^{*}$ & $0.96^{*}$ \\
\hline VAP & & & & & & & 1.00 & 0.62 & $0.81^{*}$ & $-0.82^{*}$ & $0.98^{*}$ & $0.95^{*}$ \\
\hline LIN & & & & & & & & 1.00 & $0.91^{*}$ & -0.53 & 0.61 & 0.65 \\
\hline STR & & & & & & & & & 1.00 & $-0.83^{*}$ & $0.81^{*}$ & $0.87^{*}$ \\
\hline WOB & & & & & & & & & & 1.00 & $-0.85^{*}$ & $-0.91^{*}$ \\
\hline ALH & & & & & & & & & & & 1.00 & $0.94^{*}$ \\
\hline BCF & & & & & & & & & & & & 1.00 \\
\hline
\end{tabular}

${ }^{*}$ Correlation coefficients were significant; $P<0.05$.

and between ALH with VAP, VSL, and VCL indicated that the velocity parameters were correlated and interrelated among them and with ALH. BCF was significantly and positively correlated with ALH. Anil Kumar et al. [13] and Perumal et al. [10] observed a positive correlation similar to the present study between BCF and ALH. ALH and BCF representing the head behaviour of the sperm are highly variable and mean values of these parameters were within the range. They also found a highly significant negative correlation between STR and WOB. A similar result was observed in the present study. In nonfreezable ejaculates of mithun semen, there was positive correlation between the PFM and VCL, VSL, VAP, LIN, STR, ALH, and BCF and negative correlation with SM and WOB. Similarly in freezable ejaculates, VCL is positively correlated with VSL, VAP, STR, ALH, and BCF and negatively correlated with WOB (Table 4). Similar result was reported by Anil Kumar et al. [13] and Perumal et al. [10].

Spermatozoa FPM along with certain velocity parameters are essential for the spermatozoa to achieve fertilization. Spermatozoa kinematic variables such as FPM, VSL, VCL, ALH, and LIN were correlated with bull fertility $[9,10]$. A significantly higher VCL and ALH of the spermatozoa indicate major bending of the mid piece and large amplitude of lateral head displacement. This signifies the hyperactivation of the spermatozoa. Hyperactivation in turn implies high energy state of the spermatozoa, which is essential for sperm penetration through cervical mucus and fuse with the oocytes [11]. Spermatozoa motility and velocity reflect their mitochondrial function indirectly. In bovine, specific motion parameters have been reported to be related to fertility $[7,9]$, but the threshold levels for these motion characteristics have not yet been established to meet a general consensus.

The CASA variable such as linearity or linear motility is higher indicating that spermatozoa have higher rate of fertilization potential in comparison to the total motility percentage [28] and semen samples containing such spermatozoa have higher fertility rates and pregnancy rates after artificial insemination [8]. In bovine artificial insemination industry, the minimum level of motility percentage required is 50 percent [29] and the freezable ejaculates have reached that threshold level, which shows that cryopreserved mithun semen was of acceptable quality.

Recent findings suggested that assessment of motile spermatozoa in a semen sample may not be considered as 
a reliable index for semen evaluation. The objective and quantitative measurement of other sperm motion characteristics derived from observations of individual cells assessed by CASA have been found to be more efficient in predicting semen sample's potential fertility [6]. These parameters are probably important for the progression of spermatozoa into cervical mucus and the penetration of zona pellucida of oocytes [5]. Fertilization rates of human oocytes in vitro have been shown to correlate positively with sperm velocity [30]. In bovine, sperm velocity is highly correlated with the 59-day nonreturn rate [8]. In humans, VCL and BCF were significantly higher for spermatozoa which penetrated in sperm penetration assay than for those that failed to penetrate [31]. In addition to the use of computerized technique to predict semen fertility, CASA can be a useful tool to study the effects of various in vitro procedures on sperm motility as well as phenomenon of sperm hyperactivation [32]. In present study, similar observation was reported.

The results of present study were varied with other authors. This may be due to various factors such as semen collection method, initial quality of semen, method of processing of semen for CASA, time between the collection and analysis, setup of instrument in analysing of sample, accuracy of the sample chambers and number of the chambers, and field and sperm examined to provide sufficient statistical sampling materials analysed [33].

\section{Conclusion}

It was concluded from the present study that most of the sperm motility and velocity parameters of CASA were significantly higher in freezable ejaculates in comparison to the nonfreezable ejaculates of mithun. This indicates that freezable sperm has structural stability during the freezing and thawing procedure than the nonfreezable sperm that leads to freezable sperm having higher functional sperm structures to move faster and in the forward direction. Moreover CASA system proved its usefulness in routine evaluation of mithun semen especially in frozen semen bank.

\section{Conflict of Interests}

The authors declare that there is no conflict of interests regarding the publication of this paper.

\section{References}

[1] F. J. Simoons, "Gayal or mithun," in Evolution of Domesticated Animals, I. L. Manson, Ed., pp. 34-36, Longman, London, UK, 1984.

[2] S. D. Johnston, M. V. Root-Kustritz, and P. N. S. Olson, Canine and Feline Theriogenology, WB Saunders, Philadelphia, Pa, USA, 2001.

[3] M. Iguer-ouada and J. P. Verstegen, "Evaluation of the "Hamilton thorn computer-based automated system" for dog semen analysis," Theriogenology, vol. 55, no. 3, pp. 733-749, 2001.

[4] S. T. Mortimer, "A critical review of the physiological importance and analysis of sperm movement in mammals," Human Reproduction Update, vol. 3, no. 5, pp. 403-439, 1997.
[5] J. Verstegen, M. Iguer-Ouada, and K. Onclin, "Computer assisted semen analyzers in andrology research and veterinary practice," Theriogenology, vol. 57, no. 1, pp. 149-179, 2002.

[6] D. Mortimer, Practical Laboratory Andrology, Oxford University Press, New York, NY, USA, 1994.

[7] P. R. Budworth, R. P. Amann, and R. H. Hammerstedt, "A microcomputer-photographic method for evaluation of motility and velocity of bull sperm," Journal of Dairy Science, vol. 70, no. 9, pp. 1927-1936, 1987.

[8] P. B. Farrell, G. A. Presicce, C. C. Brocektt, and R. H. Foote, "Quantification of bull sperm characteristics measured by computer-assisted sperm analysis (CASA) and their relationship to fertility," Theriogenology, vol. 49, pp. 871-879, 1998.

[9] P. B. Farrell, R. H. Foote, M. M. McArdle, V. L. TrouernTrend, and A. L. Tardif, "Media and dilution procedures tested to minimize handling effects on human, rabbit, and bull sperm for computer-assisted sperm analysis (CASA)," Journal of Andrology, vol. 17, no. 3, pp. 293-300, 1996.

[10] P. Perumal, S. Selvaraju, S. Selvakumar et al., "Effect of prefreeze addition of cysteine hydrochloride and reduced glutathione in semen of crossbred jersey bulls on sperm parameters and conception rates," Reproduction in Domestic Animals, vol. 46, no. 4, pp. 636-641, 2011.

[11] R. J. Aitken, M. Sutton, P. Warner, and D. W. Richardson, "Relationship between the movement characteristics of human spermatozoa and their ability to penetrate cervical mucus and zona-free hamster oocytes," Journal of Reproduction and Fertility, vol. 73, no. 2, pp. 441-449, 1985.

[12] P. Perumal, S. Selvaraju, S. Selvakumar et al., "Reduced glutathione and cysteine hydrochloride on sperm motility and velocity parameters of poor crossbred bull semen," International Journal of Bio-Resource and Stress Management, vol. 3, no. 2, pp. 145-151, 2012.

[13] R. Anil Kumar, M. N. Sundararaman, D. V. Patel, M. Iyue, and R. Kasiraj, "Cryopreservation of semen as a venture for conservation of wild and endangered toda Buffalo Germplasm," Buffalo Bulletin, vol. 30, no. 3, pp. 210-218, 2011.

[14] S. Koonjaenak, V. Chanatinart, S. Aiumlamai, T. Pinyopumimintr, and H. Rodriguez-Martinez, "Seasonal variation in semen quality of swamp buffalo bulls (Bubalus bubalis) in Thailand," Asian Journal of Andrology, vol. 9, no. 1, pp. 92-101, 2007.

[15] D. Kumar, A. Joshi, and S. M. K. Naqvi, "Comparative semen evaluation of Malpura and Bharat merino rams by computeraided sperm analysis technique under semi-arid tropical environment," International Journal of Animal and Veterinary Advance, vol. 2, no. 1, pp. 26-30, 2010.

[16] R. Kozdrowski, A. Dubiel, W. Bielas, and M. Dzięcioł, "Two protocols of cryopreservation of goat semen with the use of computer-assisted semen analysis system," Acta Veterinaria Brno, vol. 76, no. 4, pp. 601-604, 2007.

[17] M. L. W. J. Broekhuijse, E. Šoštarić, H. Feitsma, and B. M. Gadella, "Application of computer-assisted semen analysis to explain variations in pig fertility," Journal of Animal Science, vol. 90, no. 3, pp. 779-789, 2012.

[18] A. Domosławska, S. Zduńczyk, W. Niżański, and T. Janowski, "Assessment of semen quality in infertile dogs using computerassisted sperm analysis by the Hamilton-Thorne Semen Analyser," Bulletin of the Veterinary Institute in Pulawy, vol. 57, pp. 429-432, 2013.

[19] C. W. Palmer, S. D. Amundson, L. F. C. Brito, C. L. Waldner, and A. D. Barth, "Use of oxytocin and cloprostenol to facilitate 
semen collection by electroejaculation or transrectal massage in bulls," Animal Reproduction Science, vol. 80, no. 3-4, pp. 213223, 2004.

[20] J. K. Prasad, S. Kumar, G. Mohan, U. Shankar, and S. K. Agarwal, "Biochemical studies pertaining to freezability of cross bred bull semen," Indian Journal of Veterinary Research, vol. 8, pp. 37-40, 2000.

[21] A. V. N. Rao and Y. V. R. Rao, "Seminal characteristic and freezability of crossbred bulls," Indian Veterinary Journal, vol. 73, pp. 1086-1088, 1996.

[22] P. Perumal, Cryopreservation of bovine semen with some additives for augmenting fertility [M.V.Sc. thesis], Orissa University of Agriculture and Technology, Bhubaneswar, India, 2008.

[23] K. Sarsaifi, Y. Rosnina, M. Ariff et al., "Effect of semen collection methods on the quality of pre- and post-thawed bali cattle (Bos javanicus) spermatozoa," Reproduction in Domestic Animals, vol. 48, no. 6, pp. 1006-1012, 2013.

[24] D. Mortimer, M. A. Shu, and R. Tan, "Standardization and quality control of sperm concentration and sperm motility counts in semen analysis," Human Reproduction, vol. 1, no. 5, pp. 299-303, 1986.

[25] L. Blasco, "Clinical tests of sperm fertilizing ability," Fertility and Sterility, vol. 41, no. 2, pp. 177-192, 1984.

[26] D. P. Froman and A. J. Feltmann, "Sperm mobility: a quantitative trait of the domestic fowl (Gallus domesticus)," Biology of Reproduction, vol. 58, no. 2, pp. 379-384, 1998.

[27] D. P. Froman, A. J. Feltmann, M. L. Rhoads, and J. D. Kirby, "Sperm mobility: a primary determinant of fertility in the domestic fowl (Gallus domesticus)," Biology of Reproduction, vol. 61, no. 2, pp. 400-405, 1999.

[28] T. Cremades, J. Roca, H. Rodriguez-Martinez, T. Abaigar, J. M. Vazquez, and E. A. Martinez, "Kinematic changes during the cryopreservation of boar spermatozoa," Journal of Andrology, vol. 26, no. 5, pp. 610-618, 2005.

[29] T. Hallap, M. Håård, Ü. Jaakma, B. Larsson, and H. RodriguezMartinez, "Does cleansing of frozen-thawed bull semen before assessment provide samples that relate better to potential fertility?” Theriogenology, vol. 62, no. 3-4, pp. 702-713, 2004.

[30] E. T. Donnelly, S. E. M. Lewis, J. A. McNally, and W. Thompson, "In vitro fertilization and pregnancy rates: the influence of sperm motility and morphology on IVF outcome," Fertility and Sterility, vol. 70, no. 2, pp. 305-314, 1998.

[31] P. M. Fetterolf and B. J. Rogers, "Prediction of human sperm penetrating ability using computerized motion parameters," Molecular Reproduction and Development, vol. 27, no. 4, pp. 326-331, 1990.

[32] P. B. Farrell, R. H. Foote, M. E. Simkin, E. D. Clegg, and R. J. Wall, "Relationship of semen quality, number of sperm inseminated, and fertility in rabbits," Journal of Andrology, vol. 14, no. 6, pp. 464-471, 1993.

[33] P. Farrell, L. V. Trouern-Trend, R. H. Foote, and A. M. D. Douglas-Hamilton, "Repeatability of measurements on human, rabbit, and bull sperm by computer-assisted sperm analysis when comparing individual fields and means of 12 fields," Fertility and Sterility, vol. 64, no. 1, pp. 208-210, 1995. 

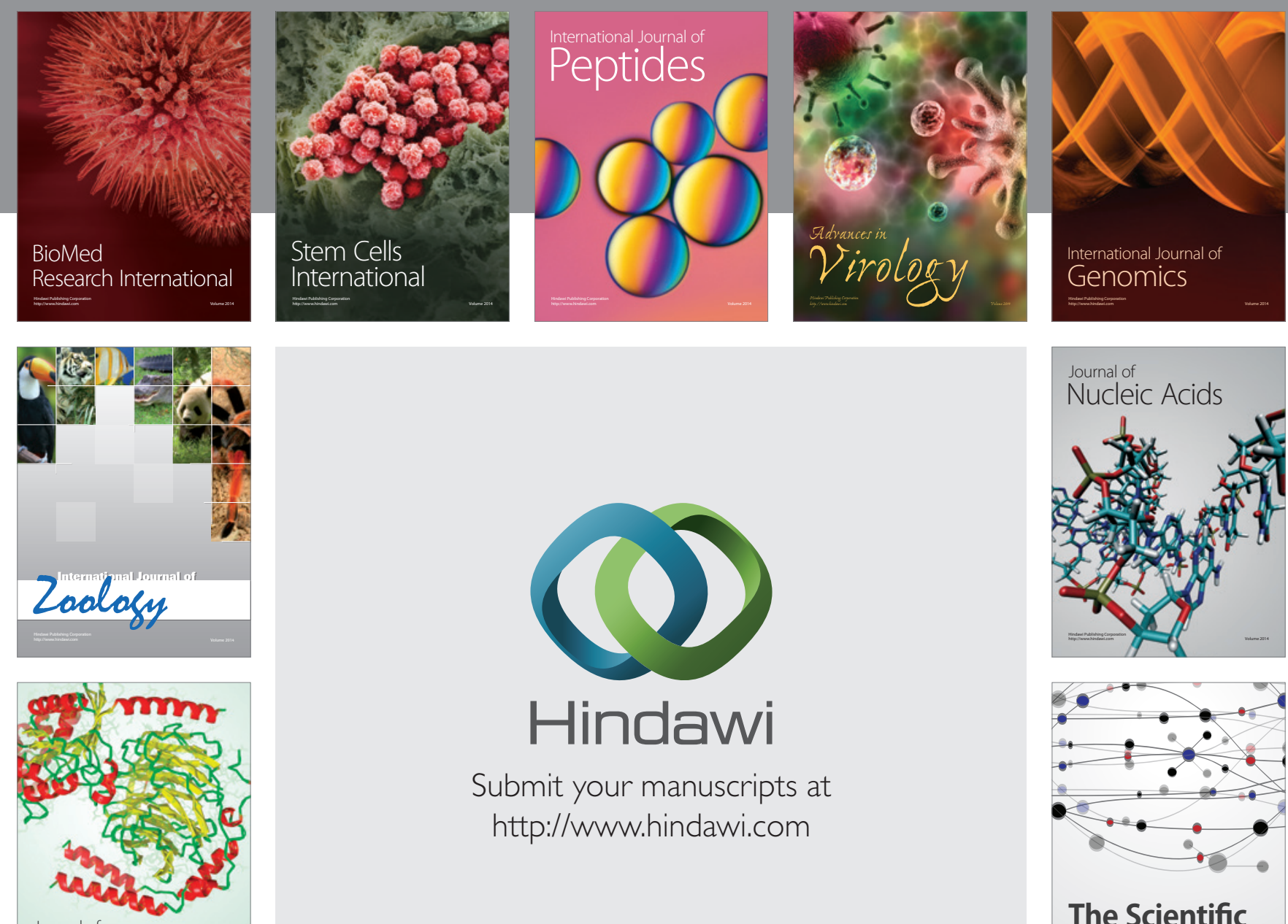

Submit your manuscripts at

http://www.hindawi.com

Journal of
Signal Transduction
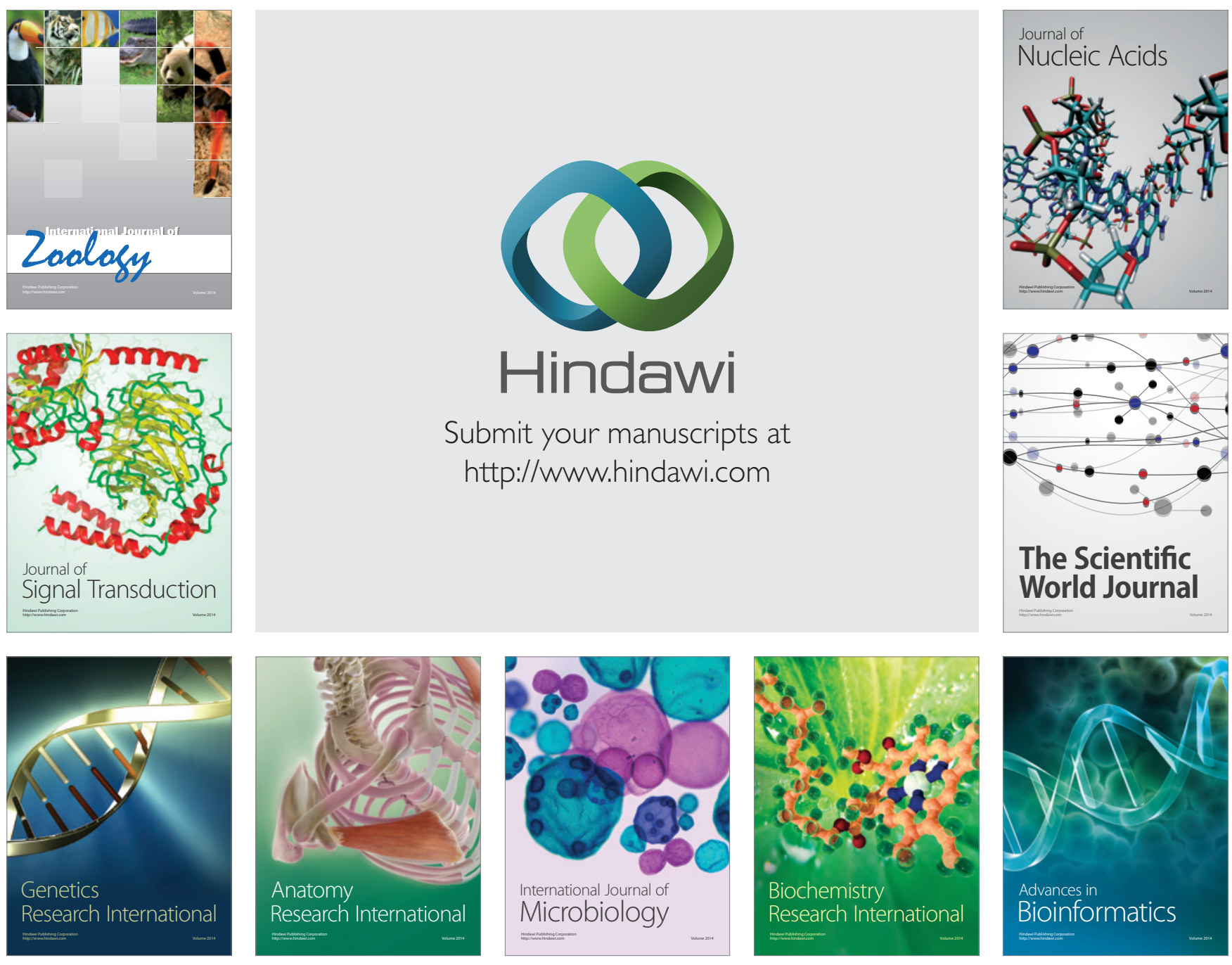

The Scientific World Journal
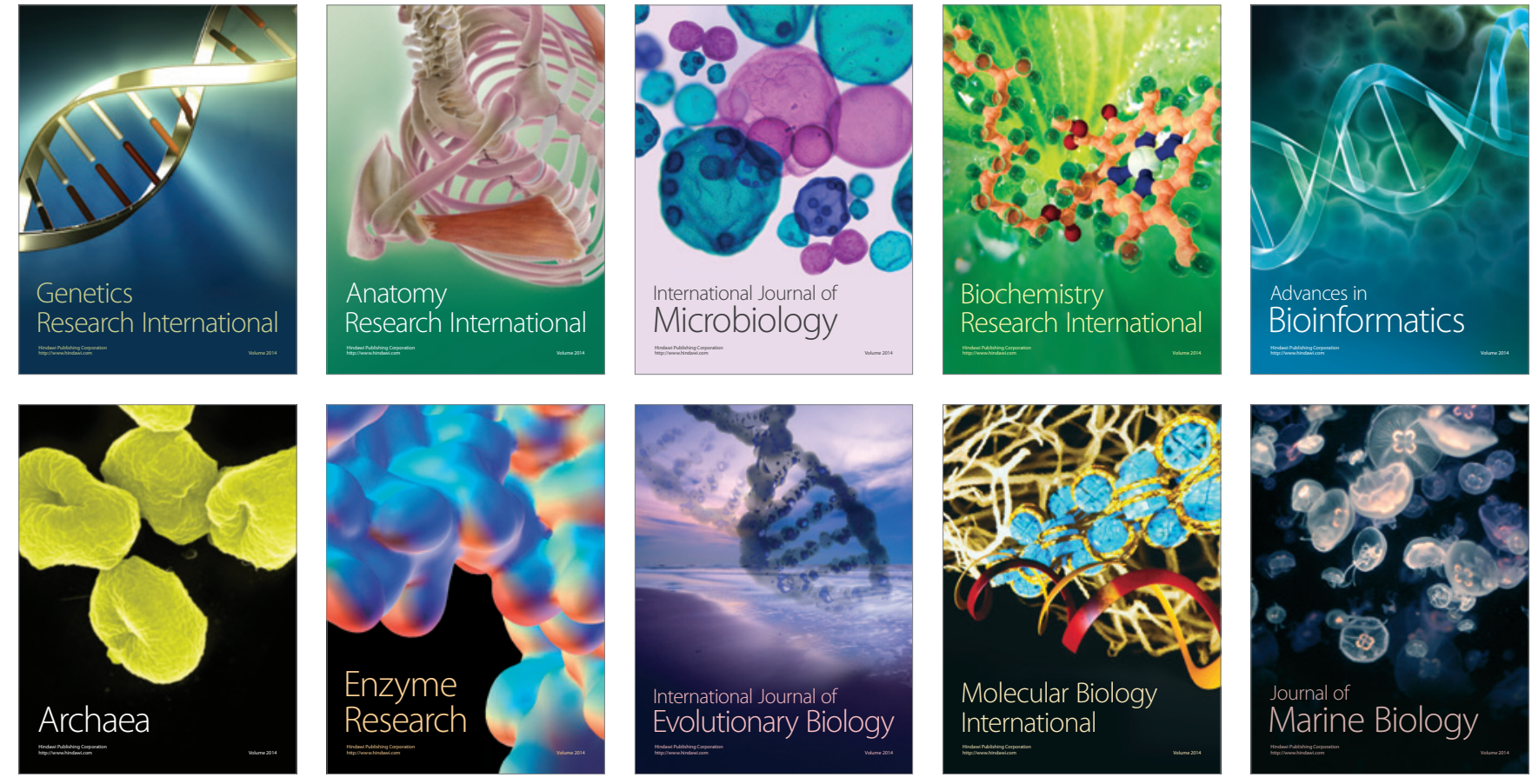